\title{
Efecto de la Paraoxonasa 1 sobre la salud y su modulación a través de la dieta
}

\section{Effect of Paraoxonase 1 on health and its modulation through diet}

\author{
Belinda P. Velázquez-Morales ${ }^{a}$, María del Carmen Valadez-Vega $^{b}$
}

\begin{abstract}
:
Paraoxonase 1 (PON1) is an enzyme expressed in liver, it circulates through bloodstream bound to high density lipoproteins (HDL). PON1 is capable to protect against cardiovascular disease development, due to its antioxidant activity. It protects HDL and low density lipoproteins (LDL) against oxidation and it detoxifies reactive molecules. It is known that high LDL serum concentration is a risk factor to develop atherosclerosis, while HDL performs an atheroprotective function, it is believed that PON1 is the main responsible of this function. Oxidative stress and inflammatory processes are capable to downregulate PON1 gene expression and enzyme activity. However, a diet rich in unsaturated fatty acids, vitamins and bioactive compounds may compensate those negative effects, upregulating PON1 expression and activity.
\end{abstract}

\section{Keywords:}

Antioxidant enzyme, enzymatic activity, protein expression, atherosclerosis, bioactive compounds.

\section{Resumen:}

La paraoxonasa 1 (PON1) es una enzima que se expresa en hígado y que circula por el torrente sanguíneo asociada a las lipoproteínas de alta densidad (HDL). La PON1 tiene la capacidad de proteger contra el desarrollo de la enfermedad cardiovascular, gracias a su actividad antioxidante. Protege a las HDL y a las lipoproteínas de baja densidad (LDL) contra la oxidación y detoxifica moléculas reactivas. Se sabe que una elevada concentración de LDL en suero es un factor de riesgo para el desarrollo de la aterosclerosis, mientras que las HDL cumplen con una función ateroprotectora, se cree que la PON1 es la principal encargada de esta función. El estrés oxidativo y los procesos inflamatorios tienen la capacidad de disminuir la expresión del gen PON1 y de la actividad de la enzima. Sin embargo, una dieta rica en ácidos grasos insaturadas, vitaminas y compuestos bioactivos puede compensar dichos efectos negativos, elevando la expresión y la actividad de la PON1.

\section{Palabras Clave:}

Enzima antioxidante, actividad enzimática, expresión de proteínas, aterosclerosis, compuestos bioactivos.

\section{Introducción}

La paraoxonasa 1 (PON1) es uno de los tres miembros de la familia de las paraoxonasas, junto con la paraoxonasa 2 (PON2) y 3 (PON3); los genes de las tres proteínas se localizan en el brazo largo del cromosoma humano 7 $(7 q 21-22)^{1}$.
La PON1 es una enzima dependiente de calcio, la cual posee dos sitios de unión de calcio, uno es importante para la estabilidad de la enzima, mientras que el otro para su actividad catalítica ${ }^{2}$. La PON1 se expresa en los hepatocitos y se encuentra asociada a las lipoproteínas de alta densidad (HDL), circula en el torrente sanguíneo unida a la Apo-A1 y la Apo $\mathrm{J}$ de las $\mathrm{HDL}^{3}$. Es la proteína más estudiada de la familia y se llama de esa manera por

\footnotetext{
a Universidad Autónoma del Estado de Hidalgo, Instituto de Ciencias de la Salud, https://orcid.org/0000-0002-6069-0774, Email: 1.n.belindavelmor@gmail.com

b Autor de Correspondencia, Universidad Autónoma del Estado de Hidalgo, Instituto de Ciencias de la Salud, https://orcid.org/0000-00016726-4113, Email: maria_valadez2584@uaeh.edu.mx
} 
su habilidad para hidrolizar el paraoxón, un metabolito tóxico del paratión ${ }^{1}$.

Sin embargo, también posee la habilidad de hidrolizar muchos otros sustratos, tales como compuestos organofosforados, arilesteres no fosforados y lactonas. La PON1 puede ser evaluada de acuerdo a sus diferentes actividades: su actividad como paraoxonasa (cuando el paraoxón es usado como sustrato), su actividad como arilesterasa (cuando es usado como sustrato un arilester no fosforado, ya sea el fenilacetato o el 4 p-nitrofenilo acetato) o su actividad como lactonasa (cuando se usa como sustrato al 5 -tiobutil butirolactona $u$ otras lactonas como la dihidrocumarina). También actúa sobre otros compuestos organofosforados (clorpirifos, diazinón, sarín y somán) y diversas lactonas. Debido a que actúa sobre una amplia gama de sustratos, sería más correcto usar el término "actividad de PON1" que puede ser mediada por diferentes sustratos. La actividad de la PON1 se puede medir a través de ensayos espectrofotométricos, así como inmunológicos utilizando anticuerpos².

En la actualidad, uno de los principales problemas en la investigación de PON1 es que no se conoce cuál es su sustrato natural, su actividad contra los organofosfatos parece ser un sustituto para su actividad fisiológica real, se cree que algunas biomoléculas endógenas con estructura de lactona de ácidos grasos poliinsaturados pueden ser los sustratos naturales de la PON $1^{4}$.

Los dos polimorfismos más importantes de la PON1 son el Q192R y el L55M. Sin embargo, el polimorfismo en la posición 192 parece estar íntimamente envuelto en las diferentes actividades de la PON1 entre individuos. Este polimorfismo tiene dos isoformas, $Q$ y $R$, dando lugar a tres fenotipos: QQ (con baja actividad enzimática), $Q R$ (con actividad enzimática intermedia) y RR (con alta actividad), lo que puede producir diferencias significativas en los valores de la actividad de PON1 entre cada individuo ${ }^{5}$.

Inicialmente, el interés sobre esta enzima fue gracias a su capacidad protectora sobre el envenenamiento por los organofosfatos; recientemente, la ciencia se ha enfocado en aspectos clínicos, tales como su rol protector en la enfermedad cardiovascular, así como su uso como biomarcador de enfermedades que envuelven principalmente tres situaciones: El estrés oxidativo, la inflamación y las hepatopatías ${ }^{6}$.

PON1 cumple un ron multifuncional, ya que actúa en diferentes vías bioquímicas, tales como la protección contra el daño oxidativo y la peroxidación lipídica, contribución en la función inmunitaria, detoxificación de moléculas reactivas, bioactivación de fármacos, modulación del estrés en el retículo endoplasmático y la regulación de la proliferación y apoptosis celular.

\section{Acción de la PON1 en enfermedad cardiovascular}

Las HDL son complejos macromoleculares heterogéneos que están inversamente correlacionados con el riesgo de padecer enfermedad cardiovascular. Además, actúan como predictores de la enfermedad cardiovascular y la mortalidad, se sugiere que la disminución del potencial antiaterogénico de las HDL puede ser causado por la alteración en la actividad de PON1 ${ }^{8}$.

La aterosclerosis es un desorden crónico de la pared arterial que comienza con la acumulación de grasa y gradualmente se forman placas ateroscleróticas. El nivel de HDL en plasma está inversamente correlacionado con la aterosclerosis, su efecto benéfico es en gran parte atribuido a su propiedad antioxidante mediada por la PON1 ${ }^{9}$.

Es bien conocida la relación entre el desarrollo de la aterosclerosis y las lipoproteínas de baja densidad (LDL), así como la función ateroprotectora de las HDL, puesto que son encargadas del transporte reverso del colesterol y también por su contenido de proteínas antioxidantes, dentro de las cuales la PON1 es la que presenta una mayor actividad antioxidante? ${ }^{9}$.

Las HDL tienen alta susceptibilidad a las modificaciones estructurales bajo diferentes condiciones, tales como la dislipidemia, el estrés oxidativo y la inflamación, todo esto induce a cambios dramáticos en su composición y la consecuente alteración en su potencial antiaterogénico. Los cambios en la estructura de las HDL pueden afectar su funcionalidad, incluyendo la actividad enzimática de la PON $1{ }^{10}$.

Las propiedades antioxidantes de la PON1 se encuentran asociadas a: La capacidad de proteger a las LDL y HDL de la oxidación, la disminución del estado oxidativo de los macrófagos, la estimulación del flujo de colesterol de los macrófagos, la disminución del estado oxidativo en las lesiones ateroscleróticas y la atenuación del desarrollo de aterosclerosis ${ }^{11}$. Se sabe que la PON1 posee actividad ateroprotectora, esto debido a su capacidad de proteger a las LDL y a las membranas celulares de la oxidación. La aterosclerosis es una condición inflamatoria que regula negativamente a la PON1, lo que se traduce en la disfunción de las $\mathrm{HDL}^{12}$. 
La oxidación de las HDL también provoca la disminución en la actividad de PON1. Los procesos inflamatorios afectan la estructura y función de las HDL, así como disminución de la concentración sérica de $\mathrm{HDL}^{10}$.

Ratones knockout PON1 presentan un doble aumento en la aterosclerosis, mientras que ratones transgénicos que sobre-expresan el gen PON1 son más resistentes al desarrollo de la aterosclerosis. La PON1 posee propiedades antiaterogénicas debido a que es capaz de hidrolizar a la homocisteína tiolactona, la cual juega un papel importante en la aterotrombosis; por otro lado, PON1 también protege contra la neurodegeneración asociada a la hiperhomocisteinemia ${ }^{13}$.

Se ha observado que los pacientes con accidente cerebrovascular presentan una concentración sérica de HDL menor que los pacientes sanos, acompañado de un decremento en la actividad de PON1, además de un estado de inflamación y de estrés oxidativo, probablemente causado por cambios estructurales en las HDL, lo cual conlleva a la disminución en la actividad de la PON1 ${ }^{1}$.

\section{Acción de la PON1 en diabetes mellitus}

La diabetes mellitus (DM) es una enfermedad caracterizada por hiperglucemia, perfil lipídico alterado, estrés oxidativo y aumento del riesgo de desarrollar enfermedad cardiovascular. La mayoría de los casos de DM en adultos pertenecen a la clasificación de diabetes tipo 2, la cual se caracteriza por presentar resistencia a la insulina e insuficiencia de las células $\beta$ pancreáticas, con su consecuente disminución de la secreción de insulina ${ }^{14}$.

Se ha demostrado que existe una asociación entre la baja actividad de PON1 y la DM, y también con el desarrollo de las complicaciones crónicas de la DM. Se sabe que la actividad de PON1 en los pacientes con DM se encuentra marcadamente reducida, esto debido a la glicación de la PON1, así como a los cambios estructurales y funcionales en las HDL causados por la dislipidemia, hiperglucemia y estrés oxidativo. La diabetes tipo 2 se encuentra asociada a una baja actividad de la PON1 y a la afectación en el metabolismo de las HDL y las LDL. La DM es una condición fisiopatológica con un intenso estrés oxidativo, las modificaciones oxidativas de las HDL pueden disminuir la actividad de $\mathrm{PON} 1^{15}$.

\section{Acción de la PON1 en enfermedad renal}

Los riñones son piezas claves en el metabolismo y excreción de toxinas urémicas, en la enfermedad renal éstas se acumulan y junto a los estados de estrés oxidativo e inflamación pueden causar alteraciones estructurales en las HDL y su consecuente reducción en la actividad de PON1. Se ha observado que la actividad de la PON1 se encuentra disminuida en pacientes con enfermedad renal crónica ${ }^{16}$.

A su vez, la modificación de PON1 por oxidación o glicación, también atenúa su actividad y conlleva disociación de las HDL. En los pacientes con hemodiálisis, la desnutrición, la inflamación y la baja actividad de la PON1 se encuentran asociadas a una alta mortalidad ${ }^{16}$.

\section{Acción de la PON1 en cáncer}

Varios autores han demostrado que la actividad de la PON1 se encuentra disminuida en pacientes que padecen diversos tipos de cáncer:

En cuanto al cáncer ginecológico, dos estudios demostraron que las pacientes con cáncer de mama presentaron niveles bajos de la concentración sérica de HDL y baja actividad de PON1 como paraoxonasa y como arilesterasa en comparación con el grupo control ${ }^{17,18}$.

La actividad como paraoxonasa y como arilesterasa de la PON1 y la concentración de HDL en suero estaban disminuidas en pacientes con cáncer colorrectal, además la actividad de la enzima se ha encontrado mayormente disminuida en aquellas personas que presentaron metástasis ${ }^{17,19,20}$. Por otra parte, sujetos con cáncer esofágico también presentaron baja actividad como arilesterasa y paraoxonasa de la enzima ${ }^{21}$.

Pacientes con cáncer de pulmón no tratado mostraron una baja actividad enzimática de la PON1, tanto como arilesterasa como paraoxonasa, y una concentración disminuida de HDL en suero; sin embargo, no hubo diferencias significativas entre la baja actividad de la enzima y el grado del tumor, ni en la presencia de metástasis $^{17}$.

\section{La PON1 modulada por la dieta}

El consumo de polifenoles provenientes de plantas y frutas eleva los niveles de antioxidantes séricos, estos antioxidantes protegen la oxidación de las LDL, así como la oxidación lipídica de las HDL a través de sus enzimas antioxidantes asociadas, tales como la PON1. La expresión de la PON1 también puede ser modulada por 
factores ambientales, incluyendo la dieta, la actividad física, ciertos fármacos y factores genéticos ${ }^{22}$.

A pesar de la fuerte relación entre la PON1 y las HDL, la concentración sérica de las HDL y la actividad de la enzima no siempre se correlacionan. Una variedad de factores no genéticos parece influenciar la actividad y los niveles séricos de la PON1. Bajo condiciones de estrés oxidativo, la actividad enzimática de la PON1 disminuye significativamente; este decremento puede ser compensado por el consumo dietario de compuestos bioactivos tales como los polifenoles, los cuales preservan la integridad de la PON1, aumentan su expresión y actividad; por otro lado, se ha observado que el consumo moderado de alcohol y de vitaminas $C$ y $E$ también elevan los niveles séricos de la PON1 ${ }^{3}$.

Se ha demostrado que la administración de un extracto acuoso de Murraya koenigii en ratones diabetizados con estretozotocina aumentó la actividad de la PON1 en suero y en hígado ${ }^{23}$; mientras que otro trabajo reportó que un extracto rico en antocianinas, tales como cianidina-3sambubiosido y cianidina-3-glucósido, de Sambucus nigra aumentó significativamente la actividad como arilesterasa de la PON1 en ratones deficientes de Apo $E^{24}$. Un estudio efectuado en ratas Wistar alimentadas con una dieta alta en fructosa y en grasas insaturadas, demostró que el jugo de Aronia melanocarpa restableció la actividad de la PON $1^{25}$.

La dieta de individuos de la zona mediterránea se ha asociado a una baja incidencia de la enfermedad cardiovascular, esto es debido a su alto contenido de vegetales y aceites de oliva virgen, ricos en ácidos grasos insaturados y compuestos fenólicos, los cuales han elevado significativamente la actividad como arilesterasa de la PON $1^{26}$. En un ensayo clínico realizado en 142 hombres y mujeres adultas que presentaban sobrepeso y obesidad, así como dislipidemia, demostró que el consumo diario de un litro de yerba mate durante 8 semanas, aumentó los niveles séricos de la $\mathrm{PON} 1^{27}$.

\section{Efecto del fruto de la granada sobre la PON1}

Algunos alimentos, especialmente las frutas, poseen altos contenidos de polifenoles que actúan como antioxidantes al donar un átomo de hidrógeno a los radicales libres cargados negativamente. Se ha demostrado que el consumo de polifenoles, sobre todo los del fruto de la granada, incrementa la expresión de la PON1 en los hepatocitos $^{3}$, se ha reportado que la capacidad antioxidante del jugo de la granada es 3 veces mayor que la del vino tinto y el té verde, siendo los polifenoles mayoritarios en este jugo los elagitaninos y las antocianinas; la punicalagina es el principal elagitanino presente en la fruta, la cual puede ser hidrolizada a ácido elágico. Se sugiere que los efectos benéficos de la granada a la salud pueden deberse a su contenido de elagitaninos, ácido elágico, ácido punícico, flavonoides, antocianidinas, antocianinas, flavonoles y flavonas estrogénicas ${ }^{22}$.

Estudios in vitro e in vivo han demostrado que el jugo de la granada reduce los niveles plasmáticos de colesterol total, triglicéridos y LDL, y aumenta los niveles de HDL y la actividad de la PON1; estas propiedades han sido atribuidas al contenido de compuestos bioactivos, los cuales probablemente son absorbidos y transportados por las $\mathrm{HDL}^{28}$.

Un estudio realizado con ratones alimentados con una dieta alta en colesterol y grasa saturada demostró que la suplementación con jugo de granada puede aumentar la expresión del gen PON1 y la actividad de la enzima ${ }^{13}$. Otro estudio llevado a cabo con mujeres diagnosticadas con síndrome coronario agudo demostró que la suplementación durante 30 días de $25 \mathrm{~g}$ de un polvo microencapsulado del jugo de la granada puede mejorar la función endotelial y reducir la hipertrigliceridemia postprandial ${ }^{8}$.

Una investigación logró demostrar que el jugo de la granada, la punicalagina, el ácido elágico y el ácido gálico fueron capaces de aumentar la expresión del gen PON1 en hepatocitos humanos $\mathrm{HuH} 7$ a través de la vía del receptor activado por proliferadores de peroxisomas $Y$ $(\text { PPARY })^{29}$.

\section{Mecanismos de acción de los compuestos bioactivos dietarios sobre la expresión del gen PON1}

Se han sugerido algunos mecanismos de acción de los polifenoles dietarios sobre el aumento en la expresión del gen PON1, tal como la regulación de Sp1 y de la proteína cinasa $C$ (PKC), a través de la vía de la proteína cinasa activada por mitógenos (MAPK); también por la interacción de la vía de la proteína de unión al elemento regulador de esteroles 2 (SREBP-2) con Sp1; así como gracias a la vía de PPARY ${ }^{29}$. 


\section{Referencias}

Kotur-Stevuljević J, Vekić J, Stefanović A, Zeljković A, Ninić A, Ivanišević J, et al. Paraoxonase 1 and atherosclerosis-related diseases. BioFactors. 2019; biof.1549.

Ceron JJ, Tecles F, Tvarijonaviciute A. Serum paraoxonase 1 (PON1) measurement: an update. BMC Vet Res. 2014;10(1):74.

3. Estrada-Luna D, Ortiz-Rodriguez MA, Medina-Briseño L Carreón-Torres E, Izquierdo-Vega JA, Sharma A, et al. Current therapies focused on high-density lipoproteins associated with cardiovascular disease. Molecules. 2018 Oct 23;23(11).

Mackness M, Mackness B. Human paraoxonase-1 (PON1): Gene structure and expression, promiscuous activities and multiple physiological roles. Gene. 2015 Aug 1;567(1):12-21.

Wysocka A, Cybulski M, P.Wysokiński A, Berbeć H, Stążka J, Zapolski T. Paraoxonase 1 activity, polymorphism and atherosclerosis risk factors in patients undergoing coronary artery surgery. J Clin Med. 2019;8(4):441.

6. Mogarekar MR, Talekar SJ. Serum lactonase and arylesterase activities in alcoholic hepatitis and hepatitis B. Indian J Gastroenterol. 2013;32(5):307-10.

7. Martinelli N, Consoli L, Girelli D, Grison E, Corrocher R, Olivieri O. Paraoxonases: ancient substrate hunters and their evolving role in ischemic heart disease. Adv Clin Chem. 2013;59:65-100.

Estrada-Luna D, Carreón-Torres E, Bautista-Pérez R, BetanzosCabrera G, Dorantes-Morales A, Luna-Luna M, et al Microencapsulated pomegranate reverts high-density lipoprotein (HDL)-induced endothelial dysfunction and reduces postprandial triglyceridemia in women with acute coronary syndrome. Nutrients. 2019;11(8).

Lou-Bonafonte JM, Gabás-Rivera C, Navarro MA, Osada J. The search for dietary supplements to elevate or activate circulating paraoxonases. Int J Mol Sci. 2017 Feb 15;18(2)

Feingold KR, Grunfeld C. Effect of inflammation on HDL structure and function. Curr Opin Lipidol. 2016;27(5):521-30.

11. Shokri Y, Variji A, Nosrati M, Khonakdar-Tarsi A, Kianmehr A, Kashi Z, et al. Importance of paraoxonase 1 (PON1) as an antioxidant and antiatherogenic enzyme in the cardiovascular complications of type 2 diabetes: Genotypic and phenotypic evaluation. Vol. 161, Diabetes Research and Clinical Practice. 2020.

Mackness M, Mackness B. Targeting paraoxonase-1 in atherosclerosis. Vol. 17, Expert Opinion on Therapeutic Targets. 2013. p. 829-37.

13.

Estrada-Luna D, Martínez-Hinojosa E, Cancino-Diaz JC, Belefant-Miller H, López-Rodríguez G, Betanzos-Cabrera G. Daily supplementation with fresh pomegranate juice increases paraoxonase 1 expression and activity in mice fed a high-fat diet. Eur J Nutr. 2018 Feb 1;57(1):383-9.

14. Wu Y, Ding Y, Tanaka Y, Zhang W. Risk factors contributing to type 2 diabetes and recent advances in the treatment and prevention. Int J Med Sci. 2014;11(11):1185-200.

15. Wu D, Wu C, Zhong Y. The association between paraoxonase 1 activity and the susceptibilities of diabetes mellitus, diabetic macroangiopathy and diabetic microangiopathy. J Cell Mol Med. 2018 Sep 1;22(9):4283-91.

16. Florens N, Calzada C, Lyasko E, Juillard L, Soulage CO. Modified lipids and lipoproteins in chronic kidney disease: A new class of uremic toxins. Toxins (Basel). 2016 Dec 1;8(12).

17. Balci H, Genc H, Papila C, Can G, Papila B, Yanardag H, et al. Serum lipid hydroperoxide levels and paraoxonase activity in patients with lung, breast, and colorectal cancer. J Clin Lab Anal. 2012;26(3):155-60.

18. Bobin-Dubigeon C, Jaffré I, Joalland MP, Classe JM, Campone M, Hervé M, et al. Paraoxonase 1 (PON1) as a marker of short term death in breast cancer recurrence. Clin Biochem. 2012;45(16-17):1503-5

19. Ahmed NS, Shafik NM, Elraheem OA, Abou-Elnoeman SEA Association of paraoxonase-1(Q192R and L55M) gene polymorphisms and activity with colorectal cancer and effect of surgical intervention. Asian Pacific J Cancer Prev. 2015;16(2):803-9.

20. Bulbuller N, Eren E, Ellidag HY, Oner OZ, Sezer C, Aydin O, et al. Diagnostic value of thiols, paraoxonase 1 , arylesterase and oxidative balance in colorectal cancer in human. Neoplasma. 2013;60(04):419-24.

21. Sehitogullari A, Aslan M, Sayir F, Kahraman A, Demir H. Serum paraoxonase-1 enzyme activities and oxidative stress levels in patients with esophageal squamous cell carcinoma. Redox Rep. 2014;19(5):199-205.

22. Chistiakov DA, Melnichenko AA, Orekhov AN, Bobryshev Y V Paraoxonase and atherosclerosis-related cardiovascular diseases. Biochimie. 2017 Jan 1:132:19-27.

23. Saha A, Mazumder S. An aqueous extract of Murraya koenigii leaves induces paraoxonase 1 activity in streptozotocin induced diabetic mice. Food Funct. 2013;4(3):420-5.

24. Farrell N, Norris G, Lee SG, Chun OK, Blesso CN. Anthocyaninrich black elderberry extract improves markers of HDL function and reduces aortic cholesterol in hyperlipidemic mice. Food Funct. 2015 Apr 1;6(4):1278-87.

25. Francik R, Krośniak M, Sanocka I, Bartoń H, Hebda T, Francik $\mathrm{S}$. Aronia melanocarpa treatment and antioxidant status in selected tissues in wistar rats. Biomed Res Int. 2014;2014.

26. Lou-Bonafonte JM, Gabás-Rivera C, Navarro MA, Osada J. PON1 and mediterranean diet. Nutrients. 2015 May $27 ; 7(6): 4068-92$

27. Balsan G, Pellanda LC, Sausen G, Galarraga T, Zaffari D, Pontin $\mathrm{B}$, et al. Effect of yerba mate and green tea on paraoxonase and leptin levels in patients affected by overweight or obesity and dyslipidemia: A randomized clinical trial. Nutr J. 2019;18(1).

28. Rom O, Volkova N, Jeries H, Grajeda-Iglesias C, Aviram M. Exogenous (Pomegranate Juice) or Endogenous (Paraoxonase1) Antioxidants Decrease Triacylglycerol Accumulation in Mouse Cardiovascular Disease-Related Tissues. Lipids. 2018 Nov $1 ; 53(11-12): 1031-41$

29. Khateeb J, Gantman A, Kreitenberg AJ, Aviram M, Fuhrman B. Paraoxonase 1 (PON1) expression in hepatocytes is upregulated by pomegranate polyphenols: A role for PPAR- $\gamma$ pathway. Atherosclerosis. 2010;208(1):119-25. 